\title{
Chinese Text Event Detection Technology Based on Improved Neural Network
}

\author{
Ying CUI ${ }^{\mathrm{a}, 1}$ and Yongchao $\mathrm{WEI}^{\mathrm{b}}$ \\ ${ }^{a}$ Southwest China Institute of Electronic Technology, Chengdu 610036, China \\ ${ }^{b}$ Civil Aviation Flight University of China, GuangHan, 618307, China
}

\begin{abstract}
With the development of deep learning, the use of neural network for text detection has been more in-depth research and more widely used. Based on this, this paper studies the Chinese text event detection technology based on improved neural network. In the research, this paper uses the flower pollination algorithm (FPA) to improve the traditional BP neural network algorithm. By optimizing the weights and thresholds of BP neural network, a Chinese text event detection method based on improved neural network is proposed. In order to verify the effect of the Chinese text event detection method based on improved neural network, this paper compares it with the natural scene text detection method, and compares the recall rate, accuracy rate and time-consuming. The results show that the accuracy rate of the natural scene text detection method is $88 \%$, and the recall rate is $73 \%$. The accuracy rate of the text detection method based on the improved neural network is $95 \%$ and the recall rate is $86 \%$. The $\mathrm{F}$ value of the natural scene text detection method in the Chinese text event detection test is 0.79 , which takes $4.56 \mathrm{~s}$ The value of $\mathrm{F}$ in is 0.90 , which takes $0.64 \mathrm{~s}$. Therefore, the Chinese text event detection method based on improved neural network has better performance.
\end{abstract}

Keywords. Improved Neural Network, Text Event Detection, Natural Scene Text Detection, Flower Pollination Algorithm

\section{Introduction}

BP neural network is an artificial neural network model, since it was proposed, it has been widely used in various detection [1-2]. We know that BP neural network is a kind of multi-level neural network. The topological structure of BP neural network is mainly composed of three parts: input layer, hidden layer and output layer. Among them, the hidden layer has at least one layer. Between layers, neurons are fully connected. Except for the input layer, the input of each layer is closely related to the output of the previous layer [3-4]. Generally speaking, BP neural network is a kind of negative feedback neural network. It adjusts the weight through negative error response to make it closer to the nonlinear function. Therefore, the convergence speed of BP neural network is relatively slow [5-6].

In recent years, text detection has become a research hotspot and a challenging topic in the field of computer vision [7-8]. Tutz proposed the Logit model to estimate weights, rather than using weights that are entirely determined by distance. Automatically select the nearest neighbor by using a selection process such as lasso or

${ }^{1}$ Corresponding Author: Ying CUI, Southwest China Institute of Electronic Technology, Chengdu, 610036, China; E-mail: cuiying.cetc10@foxmail.com 
enhancement. Then, based on the concepts of estimation and selection, the predictor space is extended [9]. Nucci proposed an adaptive method named ASCI (Adaptive Selection of Bug Predictive Classifier), which dynamically selects a classifier that can better predict the bug propensity of a class from a set of machine learning classifiers according to the characteristics of the class. An empirical study on 30 software systems shows that ASCI performs better than five classifiers that are used alone and combined with majority voting integration[10].

In the research, this paper improves the traditional BP neural network, uses the flower pollination algorithm (FPA) to optimize the weight and threshold of the network, and proposes a Chinese text event detection method based on the improved neural network. In order to verify the effect of the improved neural network algorithm in Chinese text event detection, this paper introduces the GB2312 format data set for training, and performs fuzzy processing on the Chinese vocabulary to achieve the purpose of analyzing the meaning of the word. In this paper, experiments are conducted on the computer to compare the detection effects of natural scene text detection methods and detection methods based on improved neural networks. Accuracy rate, recall rate, $\mathrm{F}$ value and time consumption.

\section{Text Detection Algorithm and Improved BP Neural Network Text Detection Algorithm}

\subsection{Text Detection Algorithm}

Traditional text detection algorithms most of the traditional text detection algorithms are based on connected domain analysis. These algorithms first aggregate image pixels into different connected regions according to low-level features (such as light intensity, color, gradient, etc.), and then classify these connected components with classification model to filter the noise area. Traditional text detection algorithms mainly use bottomup method. These algorithms usually start with character detection or stroke detection, and then apply text detection by filtering text elements, so as to construct text lines and verify text lines. Because of this, the detection results of this method have low accuracy, poor robustness, and the implementation process is too complex. In today's rapid development of society, text detection task is facing greater challenges.

- Any length width ratio, color, font, size and shape of text may appear in the image;

- The image background is more complex and diverse, and there are a lot of texture similar to the text, which is easy to cause confusion, such as fences, vegetation and other objects;

- Image quality is uncertain, and text detection is very sensitive to image quality. The common interferences are illumination condition, motion blur, low resolution and occlusion;

- Image text distribution is dense, different texts have similar features, only in detail strokes are different, the space between multiple texts is small, some tiny details may lead to multiple texts being detected as one text. Facing these more complex and difficult challenges, the traditional multi-step text detection algorithm is easy to generate a large number of non text connected regions, which brings great difficulty to the subsequent text line splicing and text line verification. 


\subsection{Chinese Text Event Detection Based on Improved Neural Network}

The nonlinear system composed of a large number of simple computing units is neural network, which simulates the processing operation of human brain to some extent.

Since the data set of this article is mainly Chinese, it is very difficult to segment words. This article uses the red-black tree method to segment the pre-stored vocabulary in the system. When analyzing the data set, the words in the data set are segmented, and then compared with the words in the system are matched. The FPA neural network in this paper supports the GB2312 data set to achieve the ability to analyze Chinese text.

In the research, we use the error function of BP neural network as the physical state function of FPA, find the minimum value of physical function, and update the state of FPA repeatedly, that is, when the error value is the smallest, we can get the best solution of FPA. It can be used as the initial weight and threshold of BP neural network.

The global pollination process is completed by Levy flight, which is described as follows:

$$
x_{i}^{t+1}=x_{i}^{t}+L\left(x_{i}^{t}-g_{+}\right)
$$

The process of local pollination and the regularity of flowers are described as follows (2):

$$
x_{i}^{t+1}=x_{i}^{t}+\varepsilon\left(x_{j}^{t}-x_{k}^{t}\right)
$$

Where $\varepsilon$ is a random number generated from $(0,1)$ uniform distribution.

$x_{t}^{i}$ and $x_{i}^{t+1}$ in Eqs.(1) and (2) refer to the solutions of generation $\mathrm{t}$ and generation $\mathrm{t}+1$ respectively. It represents the maximum of the current population.

Optimal solution, 1 is the step parameter of Levy flight, which is calculated according to the following Eq. (3):

$$
L \sim \frac{\lambda \Gamma(\lambda) \sin \left(\frac{\pi \lambda}{2}\right)}{\pi} \frac{1}{s^{1+\lambda}}
$$

\section{Experimental Design}

Experimental preparation: This paper selects CEC corpus as the test object. CEC corpus takes the news reports of five kinds of emergencies such as earthquake, fire, road accident, terrorist attack and food poisoning as the materials. Through text preprocessing, text analysis, event marking and consistency checking, the comments are finally stored in the text.

Experimental environment: After the establishment and programming of the text event detection system, we test the system using CEC corpus to comprehensively verify the detection effect of the improved and proposed algorithm.

Programming development environment is as follows: development tools is VS2008; Development language is $\mathrm{C}++$ and STL; Operating system is Windows 7; Test text data set is 2048. The core code of the test is as follows: (Part of the code, as shown in Figure 1): 


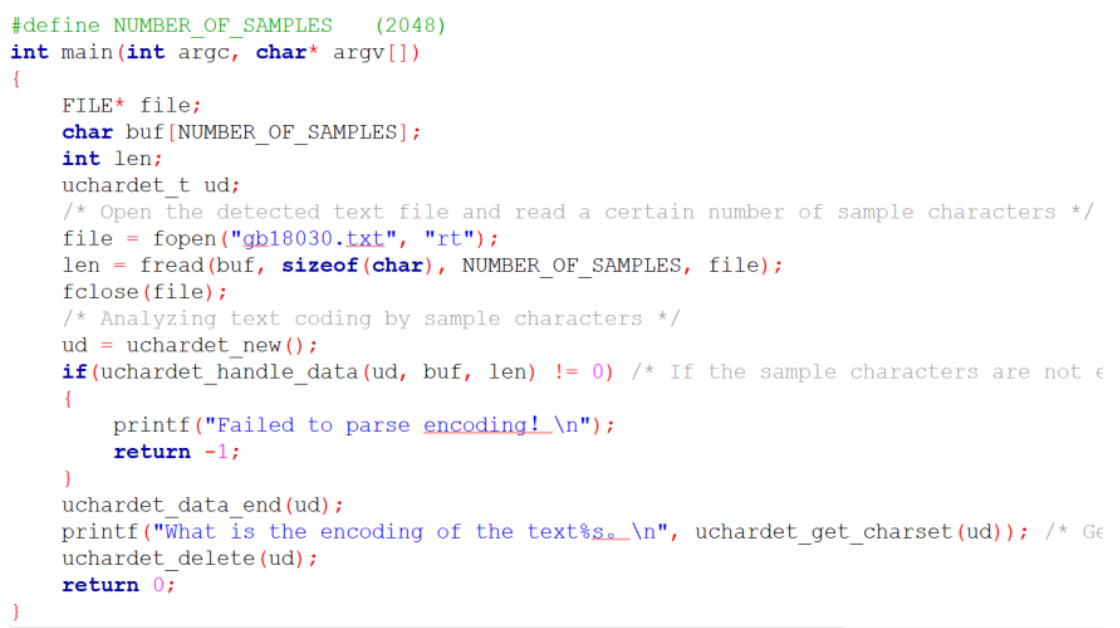

Figure 1. Text detection algorithm code

\section{Analysis and Discussion of Experimental Results}

\subsection{Data Preprocessing}

For text, neural network can not directly identify, so it is necessary to map text data into multidimensional real vector, namely text vectorization. Data preprocessing includes the following three steps.

Participle: There is no obvious boundary between words in Chinese text, so the segmentation of Chinese text is more difficult than that of English text. Whether the text can be accurately segmented has an important impact on the accuracy of text sentiment classification. "Stuttering" Chinese word segmentation is a python Chinese word segmentation library, which supports traditional word segmentation and custom dictionary. Choosing this method can segment sentences more accurately.

Word Vectorization: The distributed representation of words has very strong representation ability, and the similarity between each word can be expressed by space vector. The so-called word vectorization is to process words into the form of word vector. At present, word $2 \mathrm{vec}$ is the mainstream way to form distributed word vector. Combined with Chinese corpora, the word vector model can be obtained by using the related technology of word $2 \mathrm{vec}$, and then the word vector of each word can be obtained by inputting the word after segmentation into the vector model.

Generating word vector matrix: After word segmentation and word vectorization, a $\mathrm{N} \times \mathrm{m}$ word vector matrix will be formed. Where $\mathrm{N}$ is the number of words after word segmentation and $\mathrm{m}$ is the dimension of word vector. In order to facilitate the training of the model, it is necessary to standardize the size of the word vector matrix generated by each text, so that the size of the word vector matrix generated by all texts is the same. The processing method is:

$$
\mathrm{n}=\operatorname{avgN}+2 \times \operatorname{stdN}
$$

Among them, $\mathrm{n}$ is the number of words in the normalized word vector matrix; avgN is the average number of words $\mathrm{n} *$ after word segmentation in all texts; std $\mathrm{N}$ is the standard deviation of the number of words $n *$ after word segmentation. 
Then, the word vector matrix generated by the text is processed. The insufficient word vector matrix is filled with 0 , and the super long word vector matrix is pruned. In this way, the $\mathrm{n} \times \mathrm{M}$ word vector matrix can cover $95 \%$ of the samples.

\subsection{Selection of Common Chinese Characters}

The Chinese character sea contains 85568 Chinese characters. It can be seen that the number of Chinese characters constituting the text is quite large, but most of the Chinese characters are not commonly used. Therefore, if we take the rarely used Chinese characters and some special symbols as forbidden words and remove them from the text in the text preprocessing stage, we can improve the processing speed of the program. Therefore, this paper uses 2500 commonly used Chinese characters in modern Chinese character list to preprocess the text. Establish a common Chinese character code cross reference table, number 2500 common Chinese characters (Chinese characters are marked as w) and number them from 1 to 2500. The code of each Chinese character or the corresponding Chinese character of each code can be obtained by looking up the table. Table 1 is a partial example of the Chinese character coding Table.

Table 1. Common word coding table

\begin{tabular}{cccccccc}
\hline $\begin{array}{c}\text { Chinese } \\
\text { characters }\end{array}$ & One & Yi & Two & Ten & Ding & .. & Pot \\
\hline Number & 1 & 2 & 3 & 4 & 5 & $\ldots$ & 2500 \\
\hline
\end{tabular}

\subsection{Text Event Detection}

Whether it is Chinese text or English text, compared with other objects, characters always have their own characteristics, such as stroke width, text structure, color features of characters in images, and edge features of characters. Based on the features of the related text images, the unified induction is carried out, and the relevant decision detection rules are sorted out, so as to achieve the purpose of text event detection. Compared with the image text in the natural scene, it is easy to be affected by complex background conditions. Chinese printed text often has uniform specifications in character size, color, stroke width and texture distribution. Therefore, the printed text is detected based on the above features.

The traditional natural scene text detection algorithm basically takes English text as the detection object. Compared with the image detection of English text, the detection results of Chinese text detection are not ideal because of different styles and complex structure. In this paper, the output region of interest in Chinese printing area is combined with the improved SWT path width algorithm to detect text events. Compared with the traditional process of detecting the whole text image, the fast detection of the text region of the region of interest is undoubtedly faster.

\subsection{Analysis of Experimental Results of Chinese Text Event Detection}

(1) Analysis of accuracy and recall rate of different detection methods

This paper first analyzes the accuracy and recall rate of the two Chinese text detection methods, and the results are shown in Table 2 and Figure 1. 
Table 2. Comparison of accuracy and recall rate of different detection methods

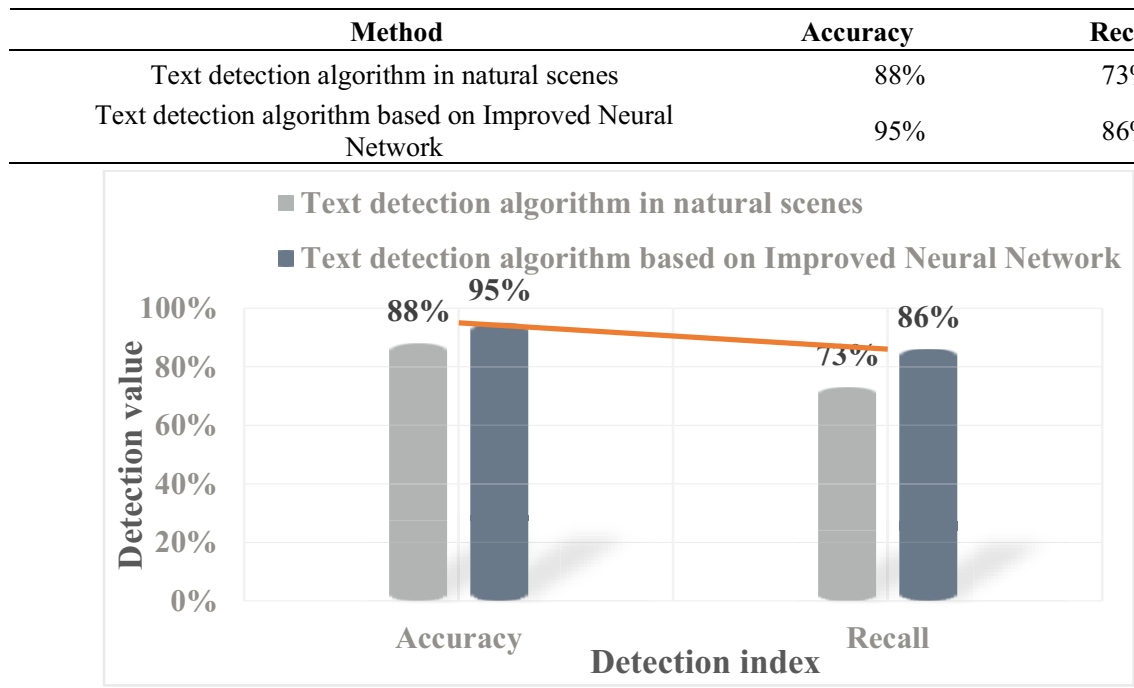

Figure 2. Comparison of accuracy and recall rate of different detection methods

It can be seen from Table 2 and Figure 2, The accuracy rate and recall rate of the two methods are different. The accuracy rate of the natural scene text detection algorithm is $88 \%$ and the recall rate is $73 \%$. The accuracy rate of the text detection algorithm based on the improved neural network is $95 \%$ and the recall rate is $86 \%$. From the comparison of the two groups of data, it can be seen that the improved neural network text detection algorithm has better performance in Chinese text event detection.

(2) F-value and time-consuming analysis of different detection methods

After analyzing the accuracy and recall rate of the two different detection algorithms, in order to further analyze the practicability of the two algorithms, this paper compares the $\mathrm{F}$ value and time consumption of the two different algorithms, and the results are shown in Table 3 and Figure 2.

Table 3. Comparison of $F$ value and time consumption of different detection methods

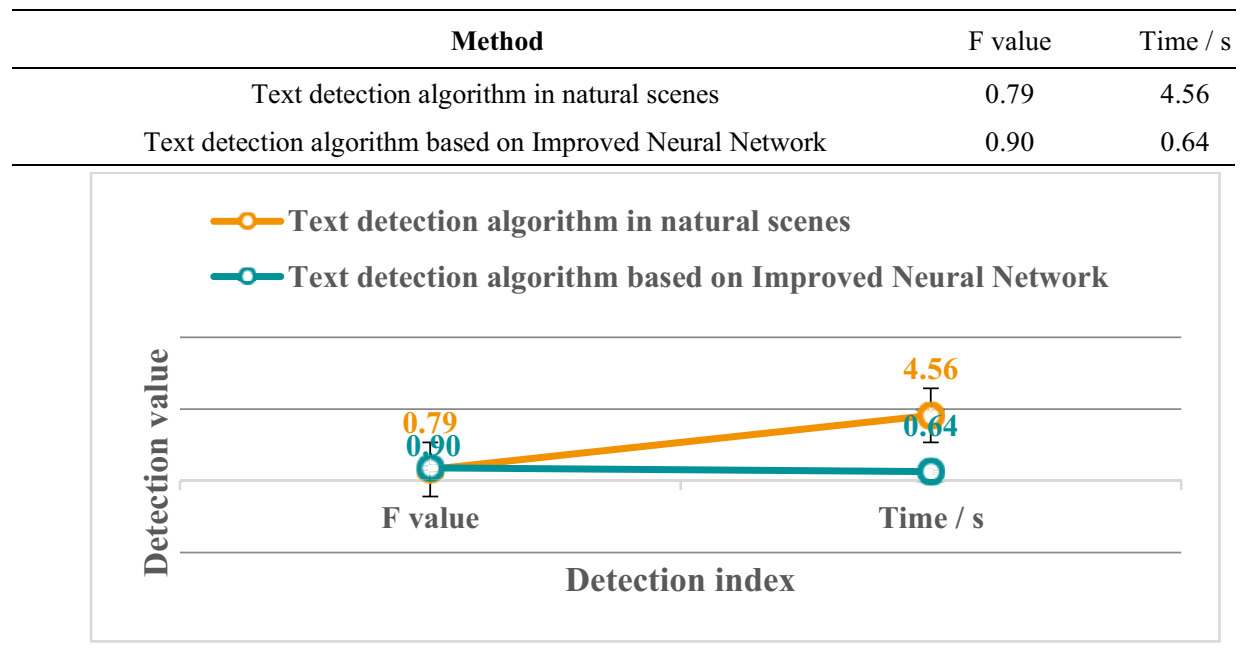

Figure 3. Comparison of $\mathrm{F}$ value and time consumption of different detection methods 
Table 3 and Figure 3 show that the $F$ value of the natural scene text detection algorithm in the Chinese text event detection test is 0.79 , which takes $4.56 \mathrm{~s}$. The $\mathrm{F}$ value of the text detection algorithm based on the improved neural network in the Chinese text event detection test is 0.90 and takes $0.64 \mathrm{~s}$. From the data comparison, it can be seen that the text detection algorithm based on the improved neural network can detect Chinese text events more quickly It is better in practicability.

\section{Conclusions}

This paper studies the Chinese text event detection technology and proposes a Chinese text event detection method based on improved neural network. In this study, we use flower pollination algorithm to optimize the weights and thresholds of the neural network. In order to verify the effectiveness of this method, we compared it with the natural scene text detection method, and analyzed the accuracy, recall rate and time consumption. The results show that the accuracy of text detection algorithm based on the improved neural network is $95 \%$ and the recall rate is $86 \%$. The F value of the text detection algorithm based on the improved neural network is 0.90 , and the time is $0.64 \mathrm{~s}$. This method has better performance. Although the text detection algorithm based on the improved neural network proposed in this paper has achieved some results, its performance is not enough to be applied to actual production. This topic will continue to conduct deep learning and optimize the algorithm in the future.

\section{References}

[1] Hu H, Wang H, Wang F, et al. Prediction of influenza-like illness based on the improved artificial tree algorithm and artificial neural network[J]. entific Reports, 2018, 8(1):4895.

[2] Huang Q H, Li B A, Lv X Q, et al. Research on Pipeline Video Defect Detection Based on Improved Convolution Neural Network[J]. Journal of Physics: Conference Series, 2020, 1576(1):012028 (6pp).

[3] Yoganand A V, Kavida A C, Rukmanidevi. Face detection approach from video with the aid of KPCM and improved neural network classifier[J]. Multimedia Tools \& Applications, 2018, 77(24):1-23.

[4] Khwaja A S, Zhang X, Anpalagan A, et al. Boosted neural networks for improved short-term electric load forecasting[J]. Electric Power Systems Research, 2017, 143(Feb.):431-437.

[5] Zhang R, Tao J. Data-Driven Modeling Using Improved Multi-Objective Optimization Based Neural Network for Coke Furnace System[J]. IEEE Transactions on Industrial Electronics, 2017, 64(4):31473155.

[6] Yang C, Pei W Y, Wu L H, et al. Chinese text-line detection from web videos with fully convolutional networks[J]. Big Data Analytics, 2018, 3(1):2.

[7] Ren X, Zhou Y, He J, et al. A Convolutional Neural Network-Based Chinese Text Detection Algorithm via Text Structure Modeling[J]. IEEE Transactions on Multimedia, 2017, PP(3):1-1.

[8] Zhu W, Lou J, Chen L, et al. Scene text detection via extremal region based double threshold convolutional network classification[J]. Plos One, 2017, 12(8):e0182227.

[9] Tutz G, Koch D. Improved nearest neighbor classifiers by weighting and selection of predictors[J]. Statistics and Computing, 2016, 26(5):1039-1057.

[10] Nucci D D, Palomba F, Oliveto R, et al. Dynamic Selection of Classifiers in Bug Prediction: An Adaptive Method[J]. IEEE Transactions on Emerging Topics in Computational Intelligence, 2017, $1(3): 202-212$. 